\title{
Adolescent and young adult cancer survivors' memory and future thinking processes place them at risk for poor mental health
}

\author{
Ursula M.Sansom-Daly, ${ }^{1,2,3,4}$ Claire E.Wakefield, ${ }^{1,2}$ Eden G.Robertson, ${ }^{1,2}$ Brittany \\ C.McGill, ${ }^{1,2}$ Helen L.Wilson, ${ }^{1,2}$ Richard A.Bryant. ${ }^{4}$
}

\author{
Affiliations: \\ ${ }^{1}$ Behavioural Sciences Unit, Kids Cancer Centre, L1 South, Sydney Children's Hospital, \\ High St., Randwick 2031, Australia. \\ ${ }^{2}$ Discipline of Paediatrics, School of Women's/Children's Health, UNSW Medicine, \\ University of New South Wales (UNSW) Sydney, \\ ${ }^{3}$ Sydney Youth Cancer Service, Australia, \\ ${ }^{4}$ School of Psychology, UNSW Sydney.
}

*Correspondence: Dr. Ursula Sansom-Daly.T:+612 9382-3114.E:ursula@ @unsw.edu.au

KEYWORDS: adolescent; autobiographical memory; cancer; future thinking; oncology; psychological adjustment; survivorship; young adult.

WORD COUNT: 3998 (4074 including title page)

This is the author manuscript accepted for publication and has undergone full peer review but has not been through the copyediting, typesetting, pagination and proofreading process, which may lead to differences between this version and the Version of Record. Please cite this article as doi: $10.1002 /$ pon.4856

This article is protected by copyright. All rights reserved. 


\begin{abstract}
Objective: Identity formation is a key developmental milestone for adolescent and young adults (AYAs). Autobiographical memory and future-thinking are crucial cognitive processes underpinning this, which may be impacted by cancer experiences. We know little about how these processes might be related to AYAs' adjustment to cancer, quality of life (QoL), and mental health outcomes.
\end{abstract}

Methods: We examined autobiographical memory and future-thinking processes, and their relationship with mental health outcomes, among 77 AYA cancer survivors $\left(M_{\text {age }}=22.3\right.$ years, $59.7 \%$ female), compared with 62 community-based controls ( $M_{\text {age }}=23.3$ years, $50 \%$ female). Participants completed the Life Narratives Interview, Future Imaginings Task, measures assessing depression, anxiety, QoL, and cancer-related identity. We coded two facets of autobiographical thinking: thematic content, and specificity.

Results: Relative to controls, survivors recounted more negative life narratives $(p=.000)$. Survivors' memories and future lives were more health/illness-focused $(p=.000)$, and remembered past events with greater specificity $(p=.007)$ than controls. In contrast, survivors' imagined their future lives with less specificity than controls $(p=.000)$. Regression analyses highlighted that being female, greater identification as a 'cancer survivor', worse depression, and recent cancer treatment-completion, significantly predicted maladaptive autobiographical thinking processes.

Conclusions: These findings point to key modifiable cognitive processes relevant to AYAs' cancer-related adjustment and future mental health. To bolster resilience into longer-term 
survivorship, clinicians could adapt existing evidence-based, cognitive-behavioural interventions to assist AYAs to imagine future events in greater detail.

"Illness has meaning, and narrative is the language of meaning.",1,p.469

While cancer is potentially devastating at any age, cancer during the adolescent/young adult (AYA) years disrupts a critical developmental period. ${ }^{2,3}$ Identity development, and a sense of purpose in terms of future life goals, are key milestones for AYAs during this time. ${ }^{4}$ As AYAs attempt to reintegrate into 'normal life' post-treatment, many show increased mental health risks for years afterwards. ${ }^{5,6}$ Research examining predictors of poorer mental health among AYAs has focused on demographic and clinical factors, with little research examining modifiable cognitive mechanisms that may underpin adjustment. ${ }^{5}$ Cancer diagnosed during the AYA years represents a rare and unexpected event which may challenge survivors' capacity to view their futures in developmentally-normal ways. ${ }^{7}$ How AYAs make sense of this experience may influence their adaptation into survivorship. ${ }^{5}$

Autobiographical thinking processes (i.e.,thinking about one's past and future) are critical to how individuals navigate and adjust to stressful events. ${ }^{8}$ These may play an even more critical role for AYAs in the midst of identity formation. ${ }^{9,10}$ Traumatic events, like cancer, can fundamentally alter individuals' beliefs about themselves and the world, as part of a 'survival' response (e.g.,developing the belief that the world is a hostile, dangerous place). ${ }^{11}$ Such beliefs may undermine psychological adaptation if they become global, rigid, and long-lasting. ${ }^{11}$ Indeed, young people who narrate their life story as biographically 'disrupted' report significant fears that their lives will continue to be disrupted, (i.e.,plans will 
continue to be quashed). ${ }^{2}$ Many survivors perceive that they have lost their 'imagined future selves' through the cancer experience, ${ }^{12}$ with AYAs describing the 'biographical disruption' of cancer to have a multifaceted, far-reaching impact on their life stories. ${ }^{10,13}$ These experiences may lead to significant grief, anger, depression, and anxiety about the future. ${ }^{14}$ The ability to process and integrate stressful life events into a meaningful life narrative is crucial to positive adaptation, and is one of the important psychological tasks of cancer survivorship. ${ }^{15,16}$

Extensive evidence highlights the importance of autobiographical thinking processes for mental health. Two aspects consistently emerge as critical. Firstly, the content of memory and future thinking - that is, what individuals remember and imagine - is strongly linked with mood and sense of self. The Self-Memory Model highlights that individuals' sense of self and self-efficacy is determined by the content of the personal, event-specific memories that they draw upon; ${ }^{17}$ for example, a cancer patient who can recall times when they successfully coped with adversity will likely feel more confident about doing so in the future. This sense of self-efficacy facilitates the ease with which individuals can recall/imagine further scenarios consistent with that version of themselves. Consequently, the content of individuals' memories and future imaginings is strongly linked with their current selfconcept. The extent to which cancer survivors' personal memories are dominated by negative, illness-focused content is therefore likely to be important for their current wellbeing.

Secondly, the specificity of autobiographical memory/future thinking is critical. Difficulty retrieving specific autobiographical memories (i.e.,event-specific 'who, where, 
what, when' details), and an inability to imagine future events in a similarly specific manner, is linked with poorer mental health across disorders. ${ }^{18}$ Non-specific, or 'overgeneral', autobiographical thinking tends to reflect a perseveration on 'category-level' memories (e.g., "All the times I broke down during chemotherapy") and has been linked with depression, ${ }^{18}$ complicated grief, ${ }^{19}$ and posttraumatic stress disorder in both children/adolescents ${ }^{20}$ and adults. ${ }^{18}$ Overgeneral thinking appears to result from rumination, reduced executive resources (the mind preoccupied by repetitive, ruminative/worry-type thinking), and emotional avoidance (avoiding distress contained in the memory's details). ${ }^{18}$ Overgeneral autobiographical thinking is a risk factor for psychological difficulties following an adverse life event. ${ }^{18}$ Little research has investigated these processes in cancer patients, though one study showed more overgeneral autobiographical thinking among head-and-neck cancer survivors with Acute Stress Disorder. ${ }^{21}$

The autobiographical systems underpinning memory and future imaginings are both thought to rely on similar underlying neural and cognitive mechanisms. ${ }^{22}$ The content and specificity of autobiographical memories appears to drive future imaginings with similar qualities. $^{23}$ This means that the adaptive processes of picturing future life events consistent with one's goals, ${ }^{17,23}$ and engaging in effective future-oriented problem-solving, ${ }^{24}$ rely upon the process of drawing specific information from memories. Indeed, integrating illness experiences into one's identity is associated with greater independence, self-management and a capacity to re-engage in activities in the chronic-illness setting. ${ }^{25}$ For AYAs on the cusp of a lifetime of survivorship, these psychological skills appear fundamental. ${ }^{5}$ 
This study examined how AYAs remember recount their life narrative, and how they imagine their future lives. We aimed to examine: whether and how AYA survivors' autobiographical past (memory) and future thinking processes differed from controls' (Research Question 1; RQ1); whether 'risky' memory features predicted these same features in future thinking (RQ2); whether survivors' autobiographical thinking processes impacted their quality of life (QoL; RQ3), and whether survivors' self-concepts differed from controls' (RQ4).

We hypothesised that survivors',

1. Memories/future imaginings would be more negative, illness-focused, and overgeneral than controls';

2. Memory risk features (i.e., negative, illness-focused, overgenerality) would predict similar features emerging in their future imaginings;

3. Negative, illness-focused, and overgeneral thinking would be associated with poorer QoL.

4. Self-concepts would be more negative than controls', and would be linked to greater distress and poorer QoL.

\section{METHODS}

\section{Participants}

This cross-sectional study was approved by South Eastern Sydney Local Health District Ethics Committee (Ref:12/068). We recruited eligible AYAs aged 15-40 (covering varying “AYA" age-definitions ${ }^{5}$ ) who had completed all forms of active, curative-intent 
cancer treatments (including maintenance chemotherapy) $\geq 1$ month prior, from a major metropolitan paediatric/AYA cancer service. Survivors could have experienced the diagnosis/treatment stages prior to age 15 . Advertisements were also placed on the research group's website, and through collaborating cancer-support organisations. We recruited control AYAs (15-40 years) with no cancer history using posters at UNSW Sydney. Across all recruitment avenues, participants opted-in by contacting the research team directly. All participants went into a draw to win a \$200 shopping voucher.

\section{Materials}

Participants provided written informed-consent, completed an interview, then a questionnaire.

Interview

Interviews ( 45minutes) were conducted by a clinical psychologist (USD/BM) via telephone or face-to-face.

Life Narratives Interview. ${ }^{8}$ This validated interview guides participants to identify "the seven most important events that have occurred in their lives" (script: Supplementary Materials 1). Participants wrote down seven discrete life events, ordered them chronologically, then recounted their life narrative. We also asked participants when each event happened, to gain an indication of the life 'span' they were remembering.

Future Imaginings Task. ${ }^{23}$ In this adapted task, participants imagined five specific, personally-significant future events (Supplementary Materials 2). Participants wrote down, then described, each future event chronologically, also estimating when they envisaged each might take place. 
Participants' memories/future imaginings were coded for several content aspects: valence (positive/negative), illness-relatedness (presence/absence of health/illness concerns), and post-traumatic growth. ${ }^{26} \mathrm{We}$ also coded for specificity (specific, intermediate, or overgeneral; Supplementary Materials 3). ${ }^{23}$

Self-concept task. A purposely-designed extension to the life narratives protocol was included to gauge AYAs' current self-concept, and perceived personal growth over their lifetime. At the start of the interview, participants chose five adjectives to describe themselves. Following the future imaginings task, participants returned to this list to identify whether they expected these attributes to change (positively/negatively) over the course of their lives. Two raters (ER/USD) coded attributes as positive (e.g., "confident”), neutral (e.g., “quiet”), or negative (e.g., “selfish”), with participants' self-reports used regarding personal growth (positive $\rightarrow$ negative/negative $\rightarrow$ positive growth).

\section{Measures}

Demographics. Participants' age, sex, education, and employment status was collected. Survivors' diagnosis and treatment regimen was also obtained.

Depression, Anxiety, Stress Scales-Short Form (DASS-21): ${ }^{27}$ The 7-item depression and anxiety subscales were included. ${ }^{18}$ The DASS-21 has been validated among large cancerfree adult ${ }^{28}$ and adolescent ${ }^{29}$ populations.

Survivors also completed cancer-specific measures:

Centrality of Events Scale (CES): ${ }^{30}$ The 7-item CES assesses the extent to which participants perceive a stressful event (in this case, cancer) has affected their sense of self, 
and ongoing worldview; higher scores indicate the event is more of a reference-point for their identity. It has been used in various trauma contexts. ${ }^{31}$

Impact of Cancer Scale (IOCS): ${ }^{32}$ We measured cancer-related QoL impact (higher scores indicating greater impact) using the IOCS, validated in 18-39-year-old survivors, ${ }^{32}$ and measures: Life Challenges, Body/Health, Talking With Parents, Personal Growth, Thinking/Memory Problems, Health Literacy, Socialising, Financial Problems.

Cancer-related identity: Using an adaptation of Park and colleagues' task ${ }^{33}$, participants indicated which label they most identified with ('cancer survivor', 'a victim of cancer', 'a young person living with cancer', 'a young person who had cancer once, but is fine now', 'none of the above-I try to ignore/forget about the fact that I had cancer,' 'other'). Participants also indicated on a 10-point visual analogue scale where they view themselves on the scale between patient and survivor $(0=$ patient $\rightarrow 10=$ survivor $)$.

\section{Data analysis}

We audio-recorded, transcribed verbatim and quantitatively scored interviews' content/process attributes. A second independent rater coded $20 \%$ responses, demonstrating adequate inter-rater reliability (mean $k$ reliability coefficients: $.90, .87$ and .82 , for life narratives valence, illness-relatedness, and specificity, respectively; $.82, .84$ and .79 , for future imaginings valence, illness-relatedness, and specificity).

Between-group analyses $(R Q \# 1,4)$. Analyses were conducted using the Statistical Package for the Social Sciences (SPSS) Version 24.0. Descriptive statistics (t-tests, chisquare analyses) were calculated to examine the sample's demographic composition. Bonferroni-adjusted analyses of covariance (ANCOVA) were undertaken to determine life 
narratives/future imaginings content/process main effects, controlling for participants' DASS-21 scores. Given the potential role of cognitive capacity on autobiographical thinking processes, ${ }^{34}$ secondary analyses were undertaken excluding brain/central nervous system (CNS) cancer survivors ( $n=6)$ as a proxy for severe cancer-treatment-related cognitive impacts (as treatment data from medical records was unavailable). An ANCOVA was also undertaken examining between-group differences on the timespan of AYAs' life narratives (years), including age as a covariate.

Identifying 'at-risk' survivors (RQ\#2). To identify predictors of maladaptive thinking processes among survivors, multivariable linear regressions examined the combined impact of demographic, clinical, and psychological processes on survivor adjustment. Each regression included demographics in Step 1 (sex, age, time post-treatment), distress in Step 2 (DASS-21), and illness-related perceptions/cognitions in Step 3 (CES scores, cancer-related identity). For future thinking regressions, Step 3 also included the corresponding memory feature (e.g., memory specificity predicting future thinking specificity). Multicollinearity tests undertaken at the outset ruled out problematic collinearity between predictors; variance inflation factor values were acceptable $(\leq 3)$ for all independent variables.

Link between autobiographical thinking and QoL (RQ\#3): Planned correlational analyses examined links between survivors' autobiographical thinking attributes (valence, health/illness-focus, and specificity), and QoL scores. Significant correlations were then tested in multivariable regressions including DASS-21 distress as a covariate, to check whether the relationship remained.

\section{RESULTS}

This article is protected by copyright. All rights reserved. 


\section{$\underline{\text { Participant characteristics }}$}

We recruited 139 participants ( $n=77$ cancer survivors, $n=62$ controls; Table 1 ). Males and females were equally represented, and were 22.7 years old on average $(S D=3.90)$. Controls reported higher depression $\left(F_{(1,129)}=7.742, p=.006\right)$ and anxiety $\left(F_{(1,129)}=8.489, p=.004\right)$ than survivors. Table 2 depicts cancer survivor characteristics. Most were diagnosed over the age of $15(n=37,55.2 \%)$, and perceived their current health was 'very good' or 'excellent' $(45 / 70$, 64.3\%; Supplementary Materials 4). Just over half identified as a 'cancer survivor' $(\mathrm{n}=37$, $52.8 \%)$, placing themselves $7.5 / 10$ on average on the 'survivor scale' $(S D=2.98$, range: $0=$ 'cancer patient'-10='survivor').

\section{RQ1: Do survivors' autobiographical thinking processes differ from controls?}

\section{Life Narratives Task}

Content

Most survivors identified their cancer diagnosis as a key life event $(n=60,77.9 \%)$. Forty-six survivors (59.7\%) described a cancer-related significant event within their life narrative $\geq 2$ times. Controlling for depression/anxiety symptoms, survivors recounted life narratives that were significantly more negative and illness-focused relative to controls (Table 3; Supplementary Materials 5). Survivors' life narratives demonstrated no more posttraumatic growth than did controls'.

All content and specificity effects for both life narratives and future thinking tasks (below) remained when excluding brain tumour/CNS survivors.

Specificity

This article is protected by copyright. All rights reserved. 
Survivors described significantly more specific life events relative to controls, but did not differ from controls on the number of extended/intermediate, or overgeneral memories they recalled (Table 3).

\section{Memory span}

Survivors' life narratives spanned a greater number of years than controls' $\left(M_{\text {cancer }}=12.60, S D_{\text {cancer }}=6.28 ; M_{\text {control }}=6.91, S D_{\text {control }}=14.26\right.$; Table 3). Survivors also recalled a mean of 2.5 memories 'clustered' a year either side of their diagnosis ( $\mathrm{SD}=1.98$, range $0-7)$; this was moderately correlated with being older at diagnosis $(r=.465, p=.000)$ and more recently post-treatment $(r=-.510, p=.000)$.

\section{Future Imaginings Task}

Content. Survivors' future imaginings were no more positive or negative than controls, but were significantly more illness-focused (Table 3).

Post-hoc, AYAs' future imaginings were coded according to whether they contained milestone events including marriage, starting a family, buying a house, and educational/career achievements (e.g., university graduation). Relative to controls, survivors imagined all future milestones at a similar frequency with one exception; survivors imagined starting a family significantly more often $\left(n=50,64.9 \%\right.$; vs. $n=22,35.5 \% ; F_{(1,127)}=12.045$, $p=.001)$.

\section{Specificity.}

Survivors' imagined futures were significantly more overgeneral, and less specific, than controls' (Table 3).

Span of future thinking. 
Survivors and controls did not differ on how far into the future they pictured key life events (Table 3).

\section{RQ2: Do 'risky' autobiographical memory features in survivors predict the emergence}

\section{of similar risk-features in future-oriented thinking?}

\section{Predictors of survivors' autobiographical memory features}

Being male, and identifying less as a 'cancer survivor,' significantly predicted greater life narrative specificity among survivors, accounting for approximately $20 \%$ of the variance $\left(F=5.944, p=0.005 ; R^{2}=.195 ;\right.$ Supplementary Materials 6). Survivors' depression/anxiety, CES scores, and time since treatment did not add to this model.

By contrast, survivors with worse depression, closer to treatment-completion, described significantly more negative life narratives ( 20\% variance; $F=5.876, p=0.005$; $\left.R^{2}=.193\right)$. Neither sex, age, anxiety, CES scores, nor 'survivor' identification added to this model.

Survivors' CES scores were the sole predictor of illness-focused memories; the more central survivors perceived their cancer experience to be to their life/identity, the more illness-concerned memories they described $\left(F=7.016, p=0.011, R^{2}=.123\right)$. No other factors significantly contributed.

\section{Predictors of future thinking features among survivors}

The sole predictor of the specificity of survivors' future imaginings was the specificity of their life narratives $\left(F=7.906, p=.007, R^{2}=.139\right)$. Survivors who recalled memories in greater detail also pictured their futures in a more specific manner. 
Echoing the autobiographical memory regressions, depressive symptoms and time since treatment-completion significantly predicted the content of survivors' future thinking. Survivors with worse depression described more negative future imaginings $(F=4.506$, $\left.p=.039, R^{2}=.083\right)$. Additionally, more recent cancer treatment-completion, together with worse depression, predicted survivors imaginings more illness-focussed futures ( $18 \%$ variance; $\left.F=5.357, p=.008, R^{2}=179\right)$.

\section{RQ3: How does survivors' autobiographical thinking impact their quality of life?}

Only one autobiographical memory/future thinking feature was associated with current QoL: having more health/illness-focused future thinking was associated with poorer IOCS scores $(r=-.445, p=.000)$. However, when survivors' distress was taken into account, illness-focused future thinking ceased to independently predict QoL ( $r=-.16, p>.05)$. This suggested that survivors' current distress explained the link between illness-focused future thinking and QoL (Fig.1).

\section{RQ4: Do survivors' self-concepts differ from controls'?}

Survivors described themselves using more positive $(F=9.992, p=.002)$ and fewer negative $(F=11.119, p=.001)$ words compared to controls. The groups did not differ on their expectations of these attributes changing into the future ( $p$-values $>.201)$. There were no links between the positivity/negativity of survivors' self-concepts and their distress/QoL ( $p$ values $>.056)$.

\section{DISCUSSION}

This study extended the literature examining how AYAs make sense of their cancer experiences, by examining cognitive processes known to place individuals at risk for poorer 
mental health using validated interviews. Recruiting controls enabled us to examine psychological processes in the context of what is developmentally normative. The use of the life narratives and future thinking paradigms is a critical extension of existing research, linking the AYA survivorship literature with broader clinical psychological science.

We found that AYA cancer survivors recounted life narratives, and imagined their futures, in ways that continued to be more illness-focussed than their peers. The ongoing dominance of illness concerns in survivors' autobiographical thinking fits with the longevity of fear of cancer recurrence, ${ }^{35}$ and may pose considerable risks to their ongoing sense of self, and adaptation into the future. ${ }^{17}$ Additionally, while survivors' memories were more specific, their imagined futures were more overgeneral. This same pattern has previously been shown among health-anxious AYAs without cancer. ${ }^{36}$ This paints a paradoxical picture whereby survivors may be able to vividly recollect a past dominated by illness, whilst simultaneously being unable - or unwilling - to clearly picture their seemingly bright future. The ability to specifically picture past and future events is strongly linked with adaptive problem-solving and goal-setting processes. ${ }^{17,23,24}$ Consequently, overgeneral thinking may adversely impact AYAs' psychosocial functioning if it leads to AYAs feeling cognitively 'stuck' in the past, and unable to see how they can move on.

Imagining non-specific, overgeneral futures could be an emotionally-avoidant coping response. ${ }^{37}$ For young people who have already experienced life-threatening illness, vividly imagining the future may be anxiety-provoking. Studies have pointed to the powerful loss of survivors' 'imagined futures' through cancer, ${ }^{12}$ and highlighted the challenges AYAs face in constructing new identities and goals into survivorship., ${ }^{2,13,16}$ Consistent with previous 
research, ${ }^{23}$ we highlighted that the ability to recall specific memories supported survivors' capacity to imagine specific futures. Given that overgeneral autobiographical thinking is a risk factor for later psychopathology, ${ }^{18}$ addressing these processes among young survivors may have an important preventative mental health impact.

We found that AYAs who identify more as a 'cancer survivor' (rather than 'patient') recounted less specific personal memories. This contrasts with research among older adults suggesting that the 'survivor' identity reflects more positive cancer-related adaptation, and leads to better psychological outcomes. ${ }^{38}$ Less research has explored this issue among AYAs, although it is possible that the survivor identity plays a different function for young people whose identities are still 'under development'. ${ }^{39}$ The few studies available suggest that while some AYAs feel ambivalent about the 'survivor' term, others actively reject it. ${ }^{13,39}$ Noncancer, mental health studies highlight the potential for a 'survivor' identity to negatively impact on psychological outcomes including recovery, suicidality, and hope. ${ }^{40}$ According to theoretical models, identifying as a 'cancer survivor' may lead to more non-specific autobiographical thinking if it causes individuals to engage in either emotional avoidance of, or rumination about, cancer-related reminders. ${ }^{37}$ Future studies are needed to explore this proposition.

\section{Clinical implications}

This study highlights the importance of assisting survivors to think about their lives in a more specific, less illness-focused manner. It is important to focus not only on what AYA cancer survivors imagine, but also on how they do so. Understanding these cognitive processes may assist us to tailor psychological interventions to target these points of 
vulnerability. For example, to reduce rumination of cancer-related content, evidence-based interventions involving mindfulness-based strategies ${ }^{35}$ could be tailored to help AYAs learn to respond more consciously and nonjudgmentally to maladaptive thinking. Some AYAs may also benefit from guided 'exposure' and practise concretely imagining and describing their new post-cancer futures. ${ }^{5,36}$ Beyond tailoring structured psychological interventions, multidisciplinary clinicians who have rapport with AYA survivors will be well-placed to incidentally prompt them in conversation to imagine personal events in event-specific ways that highlight the survivor's agency (e.g., an achievement/action/plan for a single day).

Our analyses pointed to several groups that may be more at risk: females, survivors experiencing worse depression, and survivors closer to treatment-completion. This echoes previous literature, in particular highlighting that distress may peak during the 'crisis' of treatment-completion. ${ }^{5}$ Targeting distress-screening and intervention efforts towards AYAs who have recently completed treatment may assist clinicians to capture AYAs at a point of heightened psychological vulnerability.

\section{Study Limitations}

Our findings must be interpreted in the context of several limitations. AYAs who chose to participate in an interview-based study may differ from those who chose not to (e.g.,distress levels). Recruiting via open advertisements meant that we were unable to calculate an 'opt-in rate', and were also unable to collect demographic data for nonparticipating AYAs. Our control group was more distressed than our survivors, although we 
accounted for this statistically. This may reflect a self-selected resilient cancer survivor sample, and/or a distressed university-student control population, some of whom were interviewed close to exams. We also did not measure fear of cancer recurrence, a concept likely to be linked with how survivors recall their cancer experiences and imagine their futures. Additionally, this study used a cross-sectional design; future longitudinal research could examine how AYAs' autobiographical thinking processes evolve over time, into survivorship.

\section{Future directions}

These findings open the way for psycho-oncology research in AYA patients/survivors to focus on key cognitive processes potentially underpinning their emotional outcomes across the cancer trajectory. Our results suggest numerous lines of inquiry. Firstly, given that our survivors were not clinically depressed/anxious in the aggregate, future studies must examine whether autobiographical thinking processes function similarly among survivors experiencing mental health disorders. Our regression analyses accounted for 10-20\% variance in survivors' autobiographical thinking, suggesting that other variables remain unaccounted for, beyond the demographic, medical and psychological factors studied here. Future research replicating these findings in larger survivor populations is needed. Although no longitudinal research has examined how survivors process their cancer experience over time, it is plausible that survivors may do much of this cognitive 'work' closer to the time of diagnosis and treatment. If this is the case, examining autobiographical thinking closer to this time may have had more predictive power. 
Future studies must also examine the link between autobiographical thinking processes, and functional behavioural outcomes such as the ability to plan and problem-solve towards life goals, and actual engagement in study, work, and other meaningful activities. Studies suggest that assisting AYAs to clearly imagine their future lives will in turn support their capacity to adaptively navigate future challenges. ${ }^{17,23,24}$ Demonstrating that supporting these processes translates to better lived outcomes for the growing AYA survivor population is a crucial next step.

\section{Conclusions}

How individuals remember their past and think about their future is fundamental to mental health, whether or not they have experienced cancer. Relative to controls, AYA cancer survivors show autobiographical thinking processes that may place them at risk for future adverse mental health outcomes, and may hamper their ability to engage in adaptive future behaviours. 


\section{REFERENCES}

1. Medved MI, Brockmeier J. Continuity amid chaos: Neurotrauma, loss of memory, and sense of self. Qualitative Health Research. 2008;18(4):469-79.

2. Grinyer A. The biographical impact of teenage and adolescent cancer. Chronic illness. 2007;3(4):265-77.

3. Pecchioni LL. Interruptions to cultural life scripts: Cancer diagnoses, contextual age, and life narratives. Research on Aging. 2012:0164027512449748.

4. Damon W, Menon J, Cotton Bronk K. The development of purpose during adolescence. Appl Dev Sci. 2003;7(3):119-28.

5. Sansom-Daly UM, Wakefield CE. Distress and adjustment among adolescents and young adults with cancer: An empirical and conceptual review. Translational Pediatrics. 2013;2(4):167-97.

6. Kwak M, Zebrack BJ, Meeske KA, Embry L, Aguilar C, Block R, et al. Trajectories of psychological distress in adolescent and young adult patients with cancer: a 1-year longitudinal study. Journal of clinical oncology. 2013;31(17):2160-6.

7. Tindle D, Denver K, Lilley F. Identity, Image, and Sexuality in Young Adults with Cancer. Semin Oncol. 2009;36(3):281-8.

8. Habermas T, de Silveira C. The development of global coherence in life narratives across adolescence: temporal, causal, and thematic aspects. Dev Psychol. 2008;44(3):707. 9. Beal SJ, Tillery R, Wu YP, Thompson AN, Pai A. Future orientation in adolescent and young adult cancer survivors and unaffected peers. Psycho Oncology. 2018;27(3):107881. 
10. Drew S. Self-reconstruction and biographical revisioning: survival following cancer in childhood or adolescence. Health. 2003;7(2):181-99.

11. Janoff-Bulman R. Shattered assumptions. New York: Simon and Schuster; 2010.

12. Little M, Paul K, Jordens CF, Sayers EJ. Survivorship and discourses of identity.

Psycho $\quad$-8ncology. 2002;11(2):170

13. Kumar AR, Schapira L. The impact of intrapersonal, interpersonal, and community factors on the identity formation of young adults with cancer: a qualitative study. Psychooncology. 2013;22(8):1753-8.

14. Sansom-Daly UM, Bryant RA, Cohn RJ, Wakefield CE. Imagining the future in health anxiety: the impact of rumination on the specificity of illness-related memory and future thinking. Anxiety, Stress, \& Coping. 2014;27(5):587-600.

15. Zebrack B, Bleyer A, Albritton K, Medearis S, Tang J. Assessing the health care needs of adolescent and young adult cancer patients and survivors. Cancer.

2006;107(12):2915-23.

16. Hubbard G, Forbat L. Cancer as biographical disruption: constructions of living with cancer. Support Care Cancer. 2012;20(9):2033-40.

17. Conway MA, Pleydell-Pearce CW. The construction of autobiographical memories in the self-memory system. Psychological Review. 2000;107(2):261-88.

18. Williams JMG, Barnhofer T, Crane C, Herman D, Raes F, Watkins E, et al. Autobiographical memory specificity and emotional disorder. Psychological Bulletin. 2007;133(1):122-48.

This article is protected by copyright. All rights reserved. 
19. Maccallum F, Bryant RA. Impaired autobiographical memory in complicated grief. Behaviour Research \& Therapy. 2010;48(4):328-34.

20. Nixon RD, Ball S-A, Sterk J, Best T, Beatty L. Autobiographical Memory in Children and Adolescents With Acute Stress and Chronic Posttraumatic Stress Disorder. Behav Change. 2013;30(3):180-98.

21. Kangas M, Henry JL, Bryant RA. A prospective study of autobiographical memory and posttraumatic stress disorder following cancer. Journal of Consulting and Clinical Psychology. 2005;73(2):293.

22. Schacter DL, Addis DR, Buckner RL. Remembering the past to imagine the future: the prospective brain. Nature Reviews Neuroscience. 2007;8:657-61.

23. Williams JMG, Ellis NC, Tyers C, Healy H, Rose G, MacLeod AK. The specificity of autobiographical memory and imageability of the future. Memory \& Cognition. 1996;24(1):116-25.

24. D'Zurilla TJ, Nezu A. A study of the generation-of-alternatives process in social problem solving. Cognit Ther Res. 1980;4:67-72.

25. Audulv $\AA$, Asplund K, Norbergh K-G. Who's in charge? The role of responsibility attribution in self-management among people with chronic illness. Patient Educ Counsel. 2010;81(1):94-100.

26. Tedeschi RG, Calhoun LG. " Posttraumatic growth: Conceptual foundations and empirical evidence". Psychological inquiry. 2004;15(1):1-18.

27. Lovibond SH, Lovibond PF. Manual for the Depression Anxiety Stress Scales. 2nd ed. Sydney: Psychology Foundation; 1995. 
28. Henry JD, Crawford JR. The short-form version of the Depression Anxiety Stress Scales (DASS-21): construct validity and normative data in a large non-clinical sample. British Journal of Clinical Psychology. 2005;2005(44):227-39.

29. Willemsen J, Markey S, Declercq F, Vanheule S. Negative emotionality in a large community sample of adolescents: the factor structure and measurement invariance of the short version of the depression anxiety stress scales (DASS -21). Stress Health. 2011;27(3):e120-e8.

30. Berntsen D, Rubin DC. The centrality of event scale: A measure of integrating a trauma into one's identity and its relation to post-traumatic stress disorder symptoms. Behav Res Ther. 2006;44(2):219-31.

31. Robinaugh DJ, McNally RJ. Trauma centrality and PTSD symptom severity in adult survivors of childhood sexual abuse. J Trauma Stress. 2011;24(4):483-6.

32. Zebrack BJ, Donohue JE, Gurney JG, Chesler MA, Bhatia S, Landier W. Psychometric evaluation of the impact of cancer (IOC-CS) scale for young adult survivors of childhood cancer. Qual Life Res. 2010;19(2):207-18.

33. Park CL, Zlateva I, Blank TO. Self-identity after cancer: "survivor", "victim", "patient", and "person with cancer". Journal of General Internal Medicine. 2009;24(2).

34. Ros L, Latorre JM, Serrano JP. Working Memory Capacity and Overgeneral Autobiographical Memory in Young and Older Adults. Aging Neuropsychol Cogn. 2009;17(1):89-107.

35. McNeil Beith J, Thewes B, Turner J, Gilchrist J, Sharpe L, Girgis A, et al. Long-term results of a phase II randomized controlled trial (RCT) of a psychological intervention 
(Conquer Fear) to reduce clinical levels of fear of cancer recurrence in breast, colorectal, and melanoma cancer survivors. J Clin Oncol. 2017;35(18_suppl):LBA10000-LBA.

36. Sansom-Daly UM, Bryant RA, Cohn RJ, Wakefield CE. Imagining the future in health anxiety: the impact of rumination on the specificity of illness-related memory and future thinking. Anxiety, Stress, \& Coping: An International Journal. 2014;27(5):587-600.

37. Williams JMG. Capture and rumination, functional avoidance, and executive control (CaRFAX): Three processes that underlie overgeneral memory. Cognition \& Emotion. 2006;20(3-4):548-68.

38. Cheung SY, Delfabbro P. Are you a cancer survivor? A review on cancer identity. J Cancer Surviv. 2016;10(4):759-71.

39. Murphy-Banks R. Survivor, It's Not for Everyone: Perceptions of the Cancer Survivor Label by Individuals Diagnosed with Cancer as Emerging Adults. J Pedagog Pluralism Prac. 2016;8(1):7.

40. Yanos PT, Roe D, Lysaker PH. The Impact of Illness Identity on Recovery from Severe Mental Illness. Am J Psychiatr Rehabil. 2010;13(2):73-93. 
Table 1. Participant characteristics

\begin{tabular}{|c|c|c|c|}
\hline & $\begin{array}{l}\text { Cancer } \\
(n=77)\end{array}$ & $\begin{array}{l}\text { Controls } \\
\qquad(n=62)\end{array}$ & $\begin{array}{c}\text { Total } \\
(N=139)\end{array}$ \\
\hline Age $M(S D) \dagger$ & $22.27(3.71)$ & $23.32(4.08)$ & $22.72(3.90)$ \\
\hline \multicolumn{4}{|l|}{$*_{n}=7$ missing } \\
\hline Females $n(\%) \dagger$ & $46(59.70)$ & $29(50.00)$ & $75(53.95)$ \\
\hline \multicolumn{4}{|l|}{$*_{n}=4$ missing } \\
\hline DASS21-Depression M $(S D) \neq$ & $5.16(6.72)$ & $8.87(8.52)$ & $6.92(7.82)$ \\
\hline$*_{n}=8$ missing & Range: $0-20$ & Range: $0-38$ & Range: $0-38$ \\
\hline DASS21-Anxiety $M(S D) \S$ & $5.16(6.72)$ & $7.45(6.95)$ & $5.83(6.20)$ \\
\hline$*_{n}=8$ missing & Range: $0-24$ & Range: $0-34$ & Range: $0-34$ \\
\hline
\end{tabular}

$\dagger$. Non-significant between-group difference $(\mathrm{p}>.05)$; $\ddagger$. Depression scores: $0-4=$ Normal, 5-6=Mild, 710=Moderate, 11-13=Severe, 14+=Extremely Severe; §. Anxiety scores: 0-3=Normal, 4-5=Mild, 67=Moderate, 8-9=Severe, 10+=Extremely Severe 
Table 2. Cancer survivor characteristics

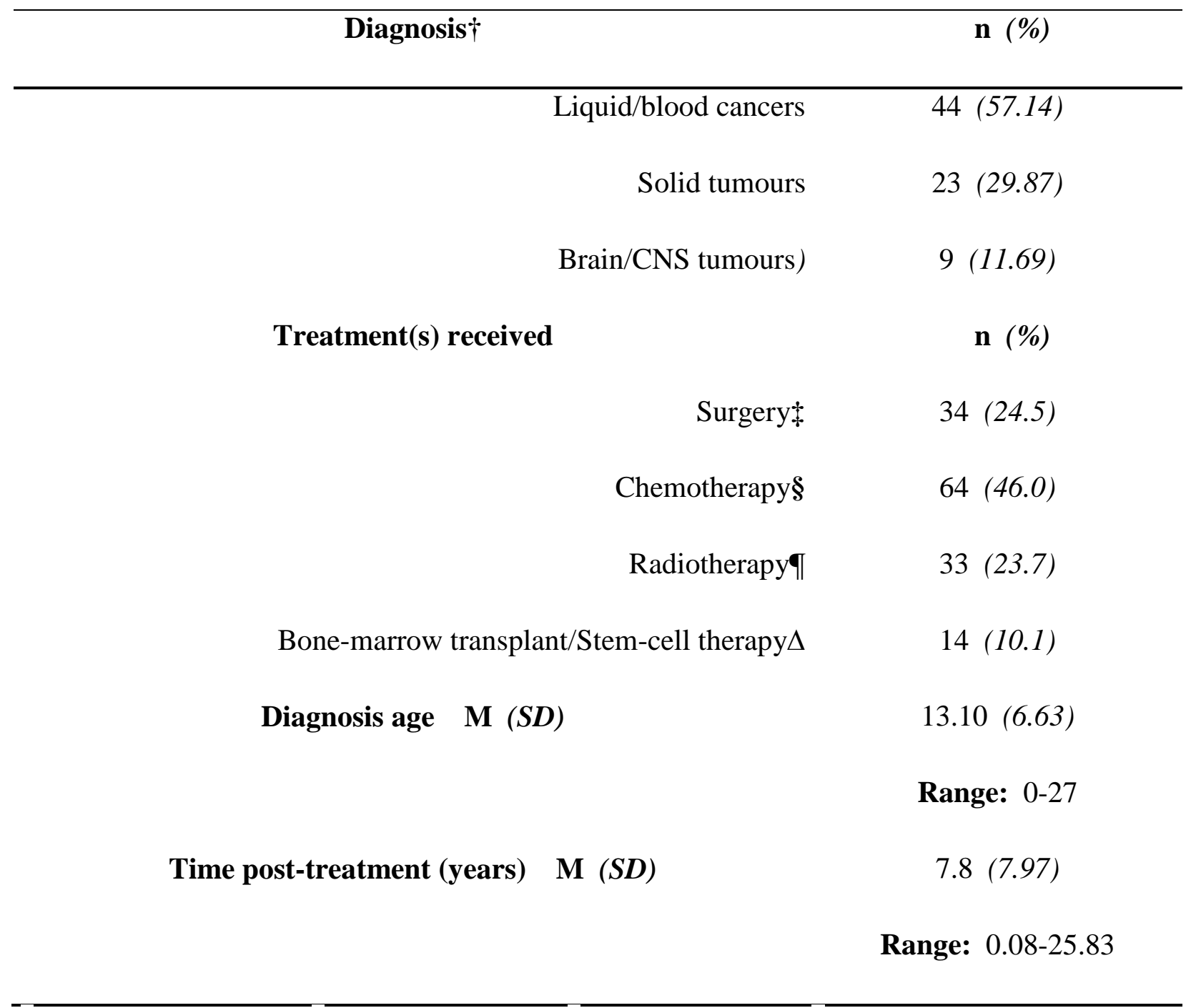

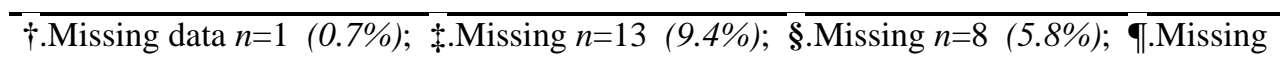
$n=11$ (7.9\%); $\Delta$.Missing $n=14$ (10.1\%)

This article is protected by copyright. All rights reserved. 
Table 3. Between-group differences in autobiographical thinking features

\begin{tabular}{|c|c|c|c|c|c|c|c|c|}
\hline \multicolumn{5}{|c|}{ Life Narratives Task } & \multicolumn{4}{|c|}{ Future Imaginings Task } \\
\hline & Control & Cancer & $F_{(1,137)}$ & Sig & Control & Cancer & $F_{(1,137)}$ & Sig \\
\hline Feature & Mean (SD) & Mean (SD) & & & Mean (SD) & Mean $(S D)$ & & \\
\hline \multicolumn{9}{|l|}{ Content } \\
\hline Positive & $4.40(1.58)$ & $4.13(1.37)$ & 1.839 & .014 & $3.87(1.26)$ & $4.25(0.88)$ & 3.846 & .052 \\
\hline Negative** & $1.50(1.25)$ & $2.26(1.18)$ & 15.695 & .000 & $0.39(0.78)$ & $0.59(0.81)$ & 2.393 & .0124 \\
\hline Illness-focused $* *$ & $0.76(1.02)$ & $3.30(1.65)$ & 108.759 & .000 & $0.58(0.97)$ & 1.32 (1.19) & 17.788 & .000 \\
\hline Post-traumatic growth & $0.19(0.44)$ & $0.41(0.75)$ & 5.660 & .019 & - & - & - & - \\
\hline \multicolumn{9}{|l|}{ Process } \\
\hline Specific** & $4.76(1.79)$ & $5.61(1.41)$ & 6.608 & .049 & $3.05(1.38)$ & $1.58(1.17)$ & 47.184 & .000 \\
\hline Intermediate/extended & $1.27(1.27)$ & $0.83(1.12)$ & 2.668 & .020 & $0.61(0.89)$ & $0.67(0.95)$ & 0.567 & .453 \\
\hline Overgeneral $* *$ & $0.69(1.00)$ & $0.52(0.88)$ & .673 & .005 & $1.19(1.14)$ & $2.72(1.29)$ & 50.252 & .000 \\
\hline Timespan (years) $* *$ & $6.91(14.26)$ & $12.59(6.28)$ & 13.605 & .000 & $6.96(26.94)$ & $16.21(26.04)$ & 2.650 & .106 \\
\hline
\end{tabular}

This article is protected by copyright. All rights reserved. 
Acknowledgements: The authors wish to acknowledge Prof Richard Cohn, Sydney

Children's Hospital, and thank Felicity Anicich, Holly Evans, Kate Marshall, Emily Spencer, Julie Ta, and Nicole Weavers, for their contributions. This work was supported by a beyond blue/Cancer Australia co-funded grant (ID:1022868), and Cancer Institute NSW (IDs:14/ECF/1-11; 11/ECF/3-43) and NHMRC grants (IDs:APP1111800; APP1067501; APP1143767). The Behavioural Sciences Unit is proudly supported by the Kids with Cancer Foundation, the Kids Cancer Alliance, and a Cancer Council NSW Program Grant PG16-02 with the support of the Estate of the Late Harry McPaul.

\section{CONFLICT OF INTEREST}

No author has any conflicts of interest to disclose in relation to this manuscript. 
Figure 1: Model showing relationship between AYA cancer survivors' illnessrelated future thinking scores, depression/anxiety scores, and quality of life

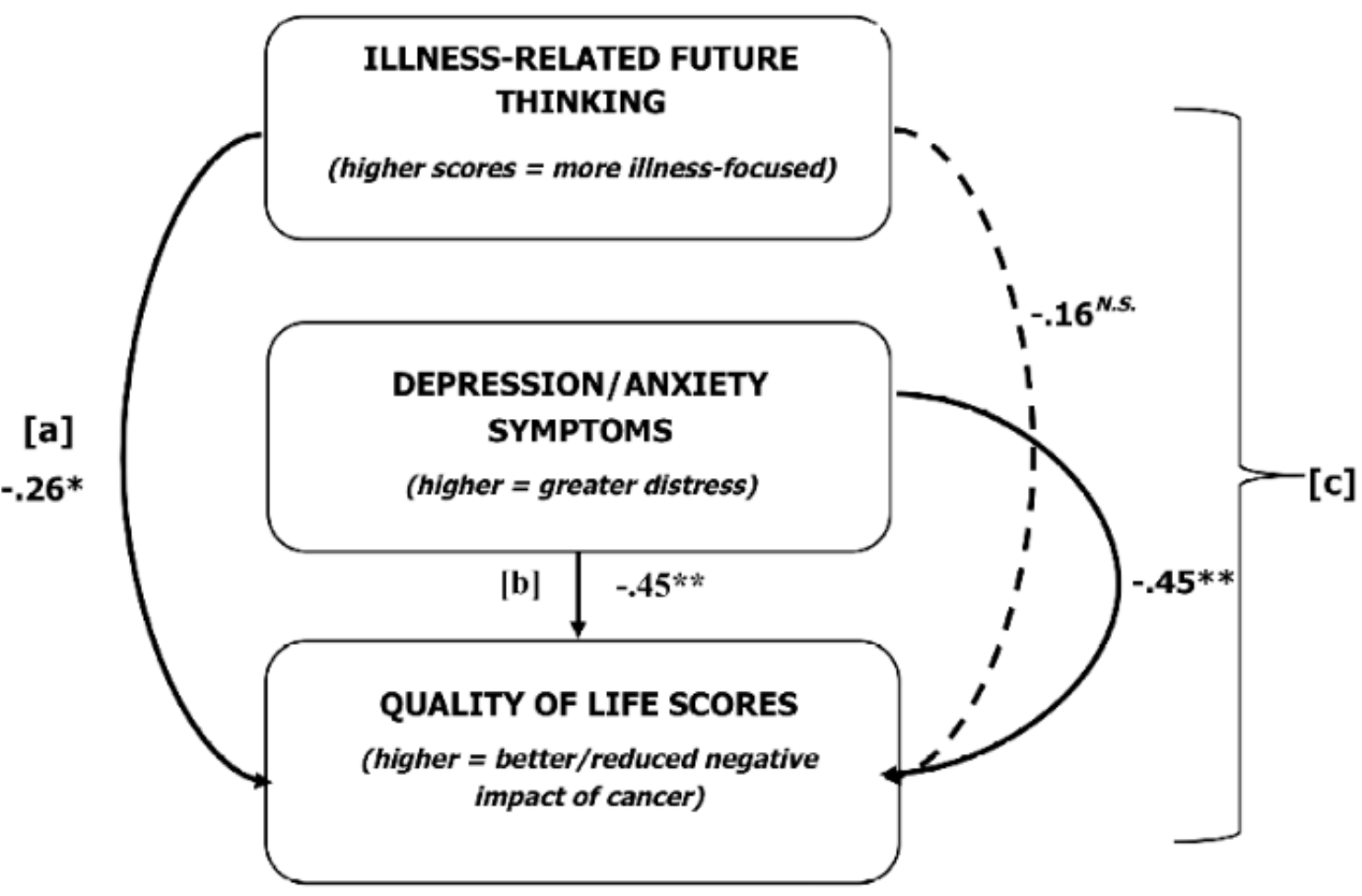

NOTE. [a] Direct negative correlation between illness-related future thinking and Impact of Cancer Scale (IOCS) quality of life impact, indicating that less illness-related future thinking was associated with a more positive (less negative) impact of the cancer experience.

[b] Direct negative correlation between depression/anxiety symptom scores and IOCS impact scores, indicating that less current depression/anxiety symptoms was associated with a greater positive (less negative) impact of cancer on quality of life.

[c] Combined relationship between illness-related future thinking, current depression/anxiety symptoms, and IOCS cancer-related impact on quality of life: indicating that when taken together, current depression/anxiety symptoms fully account for the impact on IOCS quality of life; when depression/anxiety symptoms are taken into the equation, illness-related future thinking no longer has a significant independent relationship with IOCS quality of life impact, suggesting that current distress (depression/anxiety) fully accounts for the impact on IOCS cancer-related quality of life impact. 
Figure 1: Model showing relationship between AYA cancer survivors' illnessrelated future thinking scores, depression/anxiety scores, and quality of life

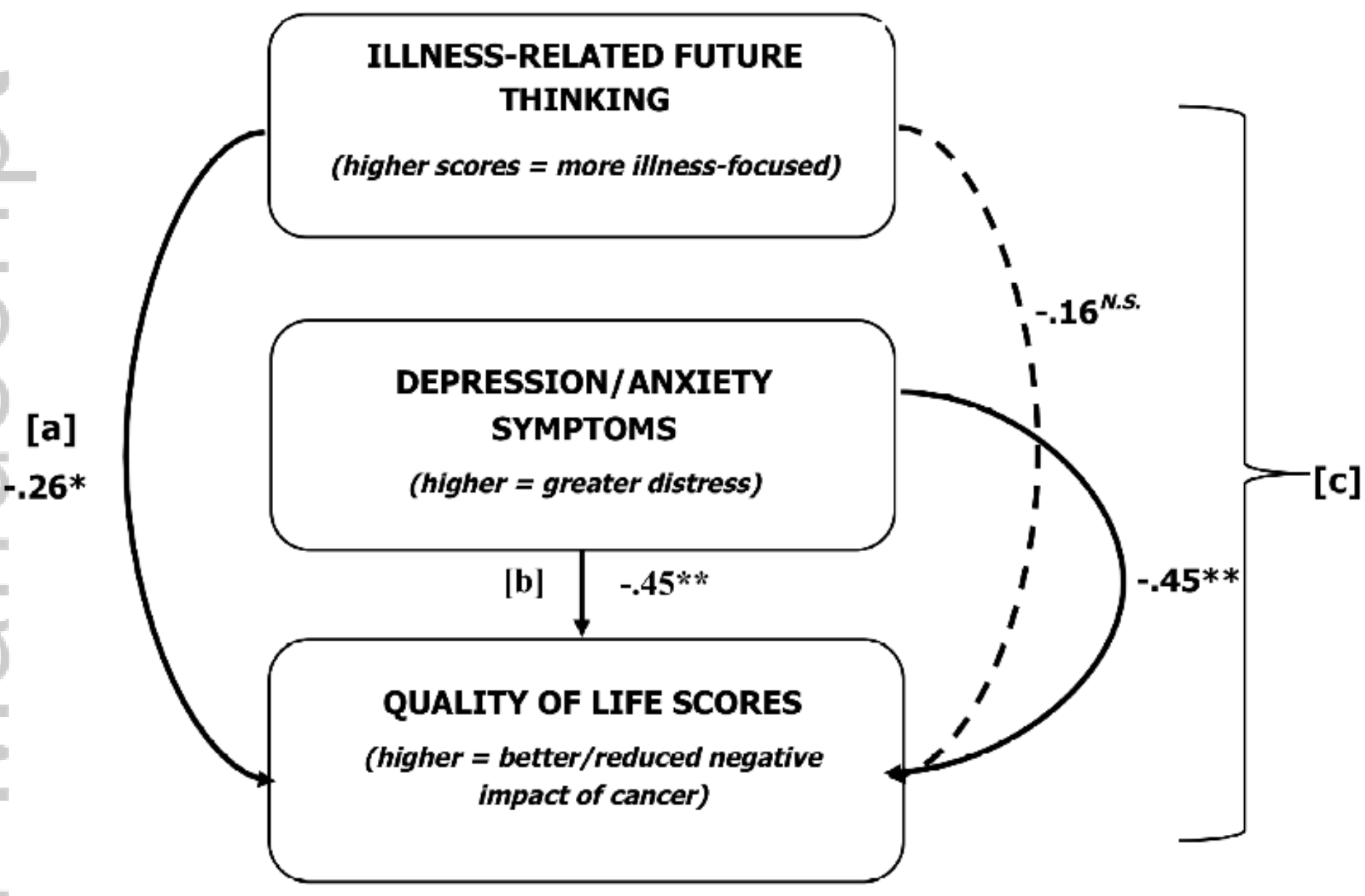

NOTE. [a] Direct negative correlation between illness-related future thinking and Impact of Cancer Scale (IOCS) quality of life impact, indicating that less illness-related future thinking was associated with a more positive (less negative) impact of the cancer experience.

[b] Direct negative correlation between depression/anxiety symptom scores and IOCS impact scores, indicating that less current depression/anxiety symptoms was associated with a greater positive (less negative) impact of cancer on quality of life.

[c] Combined relationship between illness-related future thinking, current depression/anxiety symptoms, and IOCS cancer-related impact on quality of life: indicating that when taken together, current depression/anxiety symptoms fully account for the impact on IOCS quality of life; when depression/anxiety symptoms are taken into the equation, illness-related future thinking no longer has a significant independent relationship with IOCS quality of life impact, suggesting that current distress (depression/anxiety) fully accounts for the impact on IOCS cancer-related quality of life impact. 


\section{University Library}

\section{- M M N E R VA A gateway to Melbourne's research publications}

Minerva Access is the Institutional Repository of The University of Melbourne

Author/s:

Sansom-Daly, UM;Wakefield, CE;Robertson, EG;McGill, BC;Wilson, HL;Bryant, RA

Title:

Adolescent and young adult cancer survivors' memory and future thinking processes place them at risk for poor mental health

Date:

2018-12-01

Citation:

Sansom-Daly, U. M., Wakefield, C. E., Robertson, E. G., McGill, B. C., Wilson, H. L. \& Bryant, R. A. (2018). Adolescent and young adult cancer survivors' memory and future thinking processes place them at risk for poor mental health. PSYCHO-ONCOLOGY, 27 (12), pp.2709-2716. https://doi.org/10.1002/pon.4856.

Persistent Link:

http://hdl.handle.net/11343/284619 